\title{
Anti-VEGF treatment of diabetic macular edema in clinical practice: effectiveness and patterns of use (ECHO Study Report I)
}

This article was published in the following Dove Press journal:

Clinical Ophthalmology

2I February 2017

Number of times this article has been viewed

\author{
Kevin J Blinder ${ }^{1}$ \\ Pravin U Dugel ${ }^{2,3}$ \\ Sanford Chen ${ }^{4}$ \\ J Michael Jumper ${ }^{5}$ \\ John G Walt ${ }^{6}$ \\ David A Hollander ${ }^{6}$ \\ Lanita C Scott ${ }^{6}$ \\ On behalf of the ECHO \\ Study Group \\ 'The Retina Institute, St Louis, MO, \\ ${ }^{2}$ Retinal Consultants of Arizona, \\ Phoenix, AZ, ${ }^{3}$ USC Roski Eye Institute, \\ Keck School of Medicine, University \\ of Southern California, Los Angeles, \\ ${ }^{4}$ Orange County Retina, Santa Ana, \\ ${ }^{5}$ West Coast Retina Medical Group, \\ San Francisco, ${ }^{6}$ Allergan plc, Irvine, \\ CA, USA
}

Purpose: To evaluate the efficacy, safety, and injection frequency of vascular endothelial growth factor (VEGF) inhibitors as used in clinical practice for the treatment of diabetic macular edema.

Methods: Multicenter ( 10 sites), retrospective chart review in patients ( $n=156)$ who received $\geq 3$ anti-VEGF injections. Data collected for $\geq 6$ months after the first injection included Snellen best-corrected visual acuity (BCVA) and central retinal thickness (CRT) by time-domain or spectral-domain optical coherence tomography (TD-OCT or SD-OCT).

Results: Mean number of anti-VEGF injections (627 bevacizumab, 594 ranibizumab, 1 aflibercept) was 5.8 (year 1), 5.0 (year 2), and 3.4 (year 3). Percentage of patients with BCVA of $20 / 40$ or better and CRT $\leq 250 \mu \mathrm{m}$ on TD-OCT or $\leq 300 \mu \mathrm{m}$ on SD-OCT at the same visit (primary endpoint) ranged from $16.4 \%$ to $38.9 \%$ after the first 10 injections; $51.9 \%-62.3 \%$ achieved $\geq 20 / 40$ BCVA and $26.2 \%-48.0 \%$ met CRT criteria. Therapy was well tolerated with 19 treatment-related adverse events (all ocular) reported.

Conclusion: Anti-VEGF injections were administered less frequently and were less effective than those in the ranibizumab registration trials. After each of the first 9 injections, $<25 \%$ of patients achieved both BCVA of 20/40 or better and a dry macula. A substantial proportion of patients are suboptimal responders to anti-VEGF therapy; these patients may be candidates for other therapies, including intravitreal corticosteroid and laser therapy.

Keywords: bevacizumab, diabetic retinopathy, drug administration schedule, ranibizumab, vascular endothelial growth factor, visual acuity

\section{Introduction}

Diabetic macular edema (DME) is a leading cause of vision loss in working-age adults. ${ }^{1}$ Focal and/or grid laser photocoagulation of leaking microaneurysms and areas of retinal thickening had been the standard of care for DME since the $1980 \mathrm{~s}^{2}$ However, within the past few years, intravitreal vascular endothelial growth factor (VEGF) inhibitors have emerged as preferred therapy for many patients with DME. ${ }^{3}$ The anti-VEGF treatments for DME currently approved by the United States Food and Drug Administration are ranibizumab (Lucentis; Genentech, South San Francisco, CA, USA), approved in August 2012, and aflibercept (Eylea; Regeneron Pharmaceuticals, Tarrytown, NY, USA), approved in July 2014. Bevacizumab (Avastin; Genentech) is also widely used for the treatment of DME; a formulation approved for systemic cancer therapy is usually repackaged (compounded) in syringes by a compounding pharmacy for off-label ophthalmic use and is available at lower cost. Controlled clinical studies have shown that treatment with each of these VEGF inhibitors is more effective than
Correspondence: Kevin J Blinder The Retina Institute, 1600 South Brentwood, 8th Floor, St Louis 63I44, MO, USA

$\mathrm{Tel}+|3| 4367$ | I8I

Fax + I 3149685117

Email kjblinder@gmail.com 
laser in improving vision in patients with DME. ${ }^{4-7}$ However, anti-VEGF therapy is not without limitations. The frequency of anti-VEGF injections required can be burdensome for patients. ${ }^{8,9}$ Furthermore, DME persists in some patients even after frequent anti-VEGF intravitreal injections. In the RISE/ RIDE Phase III trials of ranibizumab, DME persisted and central foveal thickness remained $>250 \mu \mathrm{m}$ on time-domain optical coherence tomography (TD-OCT) in $~ 23 \%$ of patients after 2 years of monthly ranibizumab injections. ${ }^{10}$

The recent DRCR.net Protocol T study compared the efficacy and safety of ranibizumab, bevacizumab, and aflibercept in the treatment of DME. A significant interaction was seen between baseline best-corrected visual acuity (BCVA) and the relative efficacy of the treatments in improving BCVA from baseline at year $1 .{ }^{11}$ Patients with baseline BCVA between 78 and 69 Early Treatment Diabetic Retinopathy Study (ETDRS) letters (20/32 and 20/40 Snellen equivalent) received a median of 9 ranibizumab, bevacizumab, or aflibercept injections during the year and had similar mean improvement in BCVA with each VEGF inhibitor $(+8.3,+7.5$, and +8.0 letters, respectively), while patients with baseline BCVA worse than 69 letters (20/50 Snellen equivalent or worse) received a median of 10 or 11 anti-VEGF injections during the year and had better mean improvement in BCVA at year 1 with aflibercept (+18.9 letters) than with ranibizumab (+14.2 letters) or bevacizumab (+11.8 letters). At year 2 , mean improvement in BCVA from baseline was similar with aflibercept and ranibizumab even in patients with baseline BCVA worse than 69 letters $\left(+18.1\right.$ letters and +16.1 letters, respectively). ${ }^{12}$

Among large prospective clinical trials that evaluated the efficacy and safety of ranibizumab and bevacizumab treatment in patients with DME, the largest gains in BCVA were seen in trials that used the most frequent (monthly) administration of anti-VEGF. ${ }^{13}$ These results suggest a correlation between the frequency of anti-VEGF injections and efficacy. ${ }^{13}$ As studies using medical claims data analysis suggest that ranibizumab and bevacizumab injections for DME are administered less frequently in clinical practice than in clinical trials, ${ }^{13-15}$ the efficacy of anti-VEGF treatment may be less pronounced in the clinical practice setting. Our objective in this study was to evaluate the efficacy, safety, and injection frequency of anti-VEGF therapy as used in clinical practice for the treatment of DME.

\section{Methods}

This multicenter (10 sites), retrospective, open-label study included patients who received at least 3 intravitreal injections of anti-VEGF for the treatment of DME or macular edema secondary to retinal vein occlusion (RVO). Because DME and RVO are different diseases and the use and effectiveness of anti-VEGF injections for these indications could differ, data for the DME and RVO populations underwent separate preplanned analyses. Results in the RVO population will be the subject of a second report.

The study was compliant with the International Conference on Harmonization Good Clinical Practice Guidelines and the Health Insurance Portability and Accountability Act. A central institutional review board, Sterling Institutional Review Board (Atlanta, GA, USA), approved the study protocol at each site, and all patients provided written informed consent. The study is registered at ClinicalTrials.gov with the identifier NCT01918371.

Study participants were identified between August 2013 and September 2014 by review of patient medical records from June 2010 through the time of the chart review. Patients at least 18 years of age with DME in the study eye that had been treated with at least 3 anti-VEGF intravitreal injections during this period and who had data available for a minimum of 6 months after the first anti-VEGF injection were included. Patients who had received previous intravitreal anti-VEGF treatment for DME (before June 2010), or who received the anti-VEGF injections as part of or during a clinical study, were excluded. If both eyes of a patient were eligible, the eye that had received the greater number of anti-VEGF injections was designated as the study eye.

Available data collected from medical records at the time of the first anti-VEGF injection (baseline) and through at least the next 6 months included demographics, medical and ophthalmic history, Snellen BCVA, retinal thickness in the central subfield (central retinal thickness [CRT]) by TD-OCT or spectral-domain optical coherence tomography (SD-OCT), anti-VEGF injections, concomitant treatments or procedures for DME, cataract and glaucoma surgeries, biomicroscopy/ophthalmoscopy findings, intraocular pressure (IOP), and adverse events (AEs).

The primary endpoint was the percentage of patients with both BCVA of $20 / 40$ or better and CRT $\leq 250 \mu \mathrm{m}$ on TD-OCT or $\leq 300 \mu \mathrm{m}$ on SD-OCT at the same visit. Key secondary efficacy endpoints included the percentage of patients with BCVA of 20/40 or better, the percentage of patients with CRT $\leq 250 \mu \mathrm{m}$ on TD-OCT or $\leq 300 \mu \mathrm{m}$ on SD-OCT, mean BCVA, mean change in BCVA from baseline, mean change in BCVA from baseline across all anti-VEGF injections, the percentage of patients with gain ( $\geq 1, \geq 2$, or $\geq 3$ lines), loss ( $\geq 1$ or $\geq 2$ lines), or no change ( $<1$ line change) in BCVA, mean CRT, and mean change in CRT from baseline. Other key endpoints included the mean time between anti-VEGF injections, the mean number of anti-VEGF injections each 
study year, the percentage of patients who were switched among different anti-VEGF agents, and the percentage of patients who received focal laser or panretinal photocoagulation during the study period.

All data analyses were based on observed values with no imputation of missing values. Snellen visual acuity values were converted to approximate ETDRS line scores for analysis using the method described by Gregori et al. ${ }^{16}$ Efficacy data (BCVA and CRT) were analyzed at baseline and by anti-VEGF injection number. For each injection, the BCVA and CRT representing the greatest improvement from baseline after the injection and before the next injection (peak effect) were used for analysis. Results presented in figures are truncated after the 12th injection because of the small sample size after subsequent injections (11 patients had both BCVA and CRT data available after the 13th injection). The change in BCVA across all anti-VEGF injections was determined for each patient using the peak BCVA after each injection and an area-under-the-curve approach. Changes in BCVA and CRT from baseline were evaluated with paired $t$-tests. The statistical analysis used SAS version 9.4 software (SAS Institute Inc, Cary, NC, USA) and a 2-sided alpha level of 0.05 .

\section{Results}

\section{Patient population}

Medical records of 156 patients who received $\geq 3$ anti-VEGF injections to treat DME in the study eye were reviewed. Baseline characteristics of the patients are listed in Table 1. The mean age of the study population was 63.4 years, and most patients were White and diagnosed with type 2 diabetes. The median time since diagnosis of DME in the study eye was 2 weeks (range, 0-609 weeks). Glycated hemoglobin (HbA1c) data were available in the charts for approximately half the patients; most of the patients with available $\mathrm{HbAlc}$ data had good glycemic control (HbA1c $\leq 8 \%$ ) (Table 1$)$. More than half of the patients had follow-up data for 1 year or longer.

\section{Patterns of anti-VEGF use}

A total of 1,222 anti-VEGF injections were administered to the 156 patients during the study period; 627 (51.3\%) were bevacizumab and 594 (48.6\%) ranibizumab. Only 1 patient received aflibercept ( 1 injection). Figure 1 shows the mean number of anti-VEGF injections received in the first, second, and third year after the initial anti-VEGF injection for patients who had data available throughout the year and received at least 1 injection during the year. For patients who had at least 50 weeks of data available, the mean number of anti-VEGF
Table I Baseline characteristics of patients and study eyes

\begin{tabular}{|c|c|}
\hline Parameters & $\begin{array}{l}\text { Study population } \\
(n=156)\end{array}$ \\
\hline Mean age, years (SD) & $63.4(11.3)$ \\
\hline \multicolumn{2}{|l|}{ Race, n (\%) } \\
\hline White & $109(69.9)$ \\
\hline Black/African-American/Asian & $15(9.6)$ \\
\hline Not recorded & $32(20.5)$ \\
\hline \multicolumn{2}{|l|}{ Type of diabetes, $n(\%)$} \\
\hline Type I & $13(8.3)$ \\
\hline Type 2 & $126(80.8)$ \\
\hline Not recorded & $17(10.9)$ \\
\hline \multicolumn{2}{|l|}{ HbAlc, n (\%) } \\
\hline$\leq 8 \%$ & $68(43.6)$ \\
\hline $9 \%-12 \%$ & $16(10.3)$ \\
\hline$\geq 13 \%$ & $2(1.3)$ \\
\hline Not recorded & $70(44.9)$ \\
\hline \multicolumn{2}{|l|}{ Diabetic retinopathy (NPDR) severity, n (\%) } \\
\hline Mild & $22(14.1)$ \\
\hline Moderate & $55(35.3)$ \\
\hline Severe & $17(10.9)$ \\
\hline Not recorded & $62(39.7)$ \\
\hline Median time since DME diagnosis, weeks & 2 \\
\hline Mean (range) & $51(0-609)$ \\
\hline \multicolumn{2}{|l|}{ History of procedures in study eye, $n(\%)$} \\
\hline Retinal focal laser & $63(40.4)$ \\
\hline Panretinal photocoagulation & $23(14.7)$ \\
\hline Pars plana vitrectomy & $6(3.8)$ \\
\hline Intravitreal triamcinolone acetonide injection & $4(2.6)$ \\
\hline \multicolumn{2}{|l|}{ Lens status, $\mathrm{n}(\%)$} \\
\hline Phakic & $104(66.7)$ \\
\hline Pseudophakic & $51(32.7)$ \\
\hline Not recorded & $\mathrm{I}(0.6)$ \\
\hline \multirow[t]{2}{*}{ Mean BCVA, ETDRS lines (SD) } & $11.8(3.5)$ \\
\hline & $20 / 63^{-1}$ Snellen \\
\hline Mean CRT, $\mu \mathrm{m}(\mathrm{SD})$ & $413(105)$ \\
\hline
\end{tabular}

Abbreviations: BCVA, best-corrected visual acuity; CRT, central retinal thickness; DME, diabetic macular edema; ETDRS, Early Treatment Diabetic Retinopathy Study; $\mathrm{HbAlc}$, glycated hemoglobin; NPDR, nonproliferative diabetic retinopathy; SD, standard deviation.

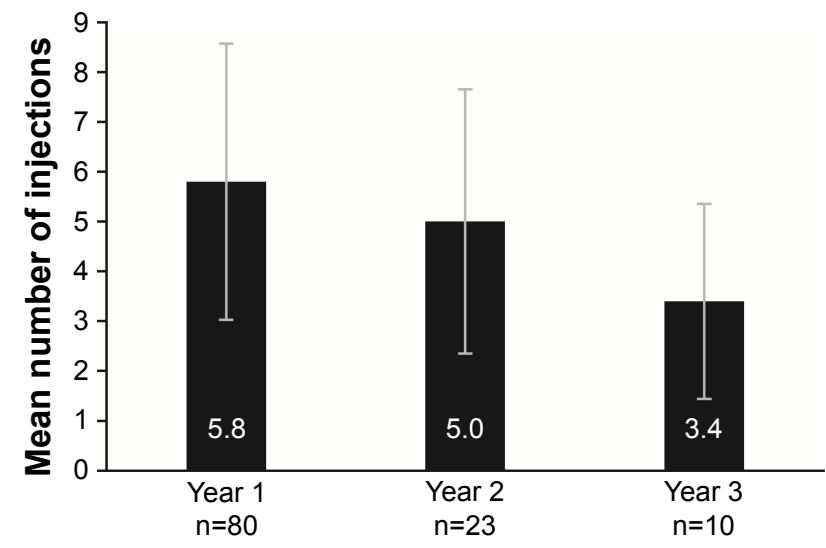

Figure I Mean number of anti-VEGF injections received by patients each study year.

Notes: To be included in the analysis, patients must have had anti-VEGF injection data for at least the following minimum time: year I, 50 weeks; year 2, 100 weeks; year 3, I50 weeks; and must have received at least I injection during the study year. Error bars indicate the standard deviation.

Abbreviation: VEGF, vascular endothelial growth factor. 
injections received during the first year of the study was 5.8 (range, 1-13) (Figure 1).

For almost half of the patients $(74 / 156,47.4 \%)$, the type of anti-VEGF therapy used was changed at least once during the study period. The most common switch in therapy was from ranibizumab to bevacizumab (70 patients, $44.9 \%$ of patients). Twenty-four (15.4\%) patients switched from bevacizumab to ranibizumab, and 1 patient switched from aflibercept to ranibizumab.

Over the course of the first 12 anti-VEGF injections, the mean time to the next injection (for patients who received another injection within the study period) ranged from 1.2 to 1.7 months (Figure 2).

\section{Primary endpoint}

Because the optimal goal of treatment is to achieve good visual acuity and a dry macula, the primary endpoint was a combined endpoint of visual acuity and anatomic response, and patients had to have both BCVA and CRT data from the same visit to be included in the analysis. After most injections, $\sim 1$ in 5 patients with analyzable data met the primary endpoint of BCVA $20 / 40$ or better and CRT $\leq 250 \mu \mathrm{m}$ on TD-OCT or $\leq 300 \mu \mathrm{m}$ on SD-OCT at the same visit (Figure 3 ). The percentage of patients who achieved the primary endpoint was $16.4 \%(17 / 104)$ after the first anti-VEGF injection and reached a maximum of $38.9 \%$ (7/18) after the tenth anti-VEGF injection. After each anti-VEGF injection, the percentage of patients who achieved 20/40 or better BCVA was generally much higher than the percentage of patients who achieved $\mathrm{CRT} \leq 250 \mu \mathrm{m}$ on TD-OCT or $\leq 300 \mu \mathrm{m}$ on SD-OCT

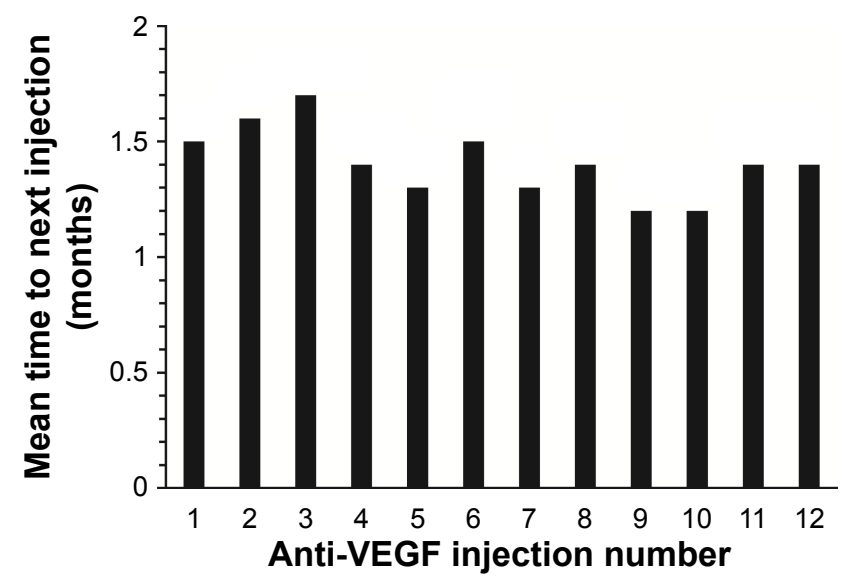

Figure 2 Mean interval between anti-VEGF injections.

Notes: Analysis of mean time to next injection was based on the number of patients who received another anti-VEGF injection within the study period. The number of patients for injection numbers I through 12 was I52, I54, I4I, I24, 106, 89, 64, 52, 40, 30, 22, and 17, respectively.

Abbreviation: VEGF, vascular endothelial growth factor.

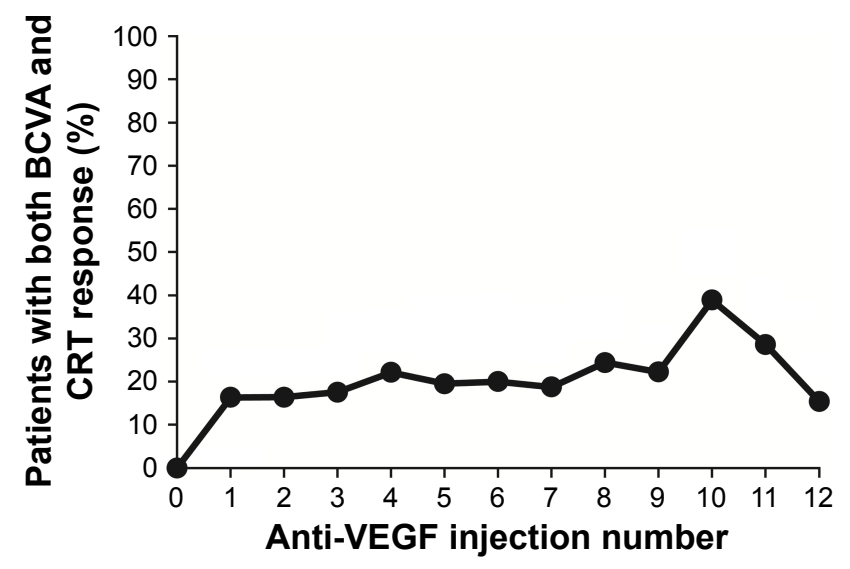

Figure 3 Percentage of patients with both BCVA and CRT response at the same visit (primary endpoint) after each anti-VEGF injection.

Notes: BCVA response was defined as $20 / 40$ or better BCVA and CRT response as CRT $\leq 250 \mu \mathrm{m}$ on TD-OCT or $\leq 300 \mu \mathrm{m}$ on SD-OCT. Percentage of patients was based on the number of patients with both BCVA and CRT measurements at the same visit. The number of patients for injection numbers 0 through 12 was 101 , 104, I10, II4, 95, 77, 70, 48, 41, 36, 18, 14, and 13, respectively.

Abbreviations: BCVA, best-corrected visual acuity; CRT, central retinal thickness; SD-OCT, spectral-domain optical coherence tomography; TD-OCT, time-domain optical coherence tomography; VEGF, vascular endothelial growth factor.

(Figure 4). After the first through ninth anti-VEGF injections, BCVA of $20 / 40$ or better was achieved by $52 \%-62 \%$ of patients, while CRT $\leq 250 \mu \mathrm{m}$ on TD-OCT or $\leq 300 \mu \mathrm{m}$ on SD-OCT was achieved by $26 \%-34 \%$ of patients.

\section{Secondary efficacy measures}

Mean change from baseline BCVA after each of the first 6 anti-VEGF injections was statistically significant $(P<0.001)$ and ranged from +1.0 to +1.3 lines (approximate ETDRS line scores; Figure 5). Across all anti-VEGF injections throughout the study period, the mean (standard deviation) change in BCVA from baseline was +1.1 (2.7) lines $(P<0.001$, area-under-the-curve approach). After the first through ninth anti-VEGF injections, the percentage of patients with a gain in BCVA from baseline of $\geq 3$ lines ranged from $14 \%$ to $29 \%$, whereas $28 \%-36 \%$ of patients had a gain in BCVA of $\geq 2$ lines and $41 \%-53 \%$ of patients had a gain in BCVA of $\geq 1$ line (Figure 6). A significant percentage of patients showed no improvement in BCVA or experienced a loss in BCVA after these injections. After the first through ninth anti-VEGF injections, $15 \%-23 \%$ of patients had a loss in BCVA from baseline of $\geq 1$ line, and $4 \%-13 \%$ of patients had a loss in BCVA from baseline of $\geq 2$ lines (Figure 6).

Mean change from baseline CRT after each of the first 10 anti-VEGF injections was statistically significant $(P \leq 0.015)$ and ranged from -45 to $-83 \mu \mathrm{m}$ (Figure $7 \mathrm{~A}$ ). Mean CRT after these injections ranged from 327 to $377 \mu \mathrm{m}$ (Figure 7B). 

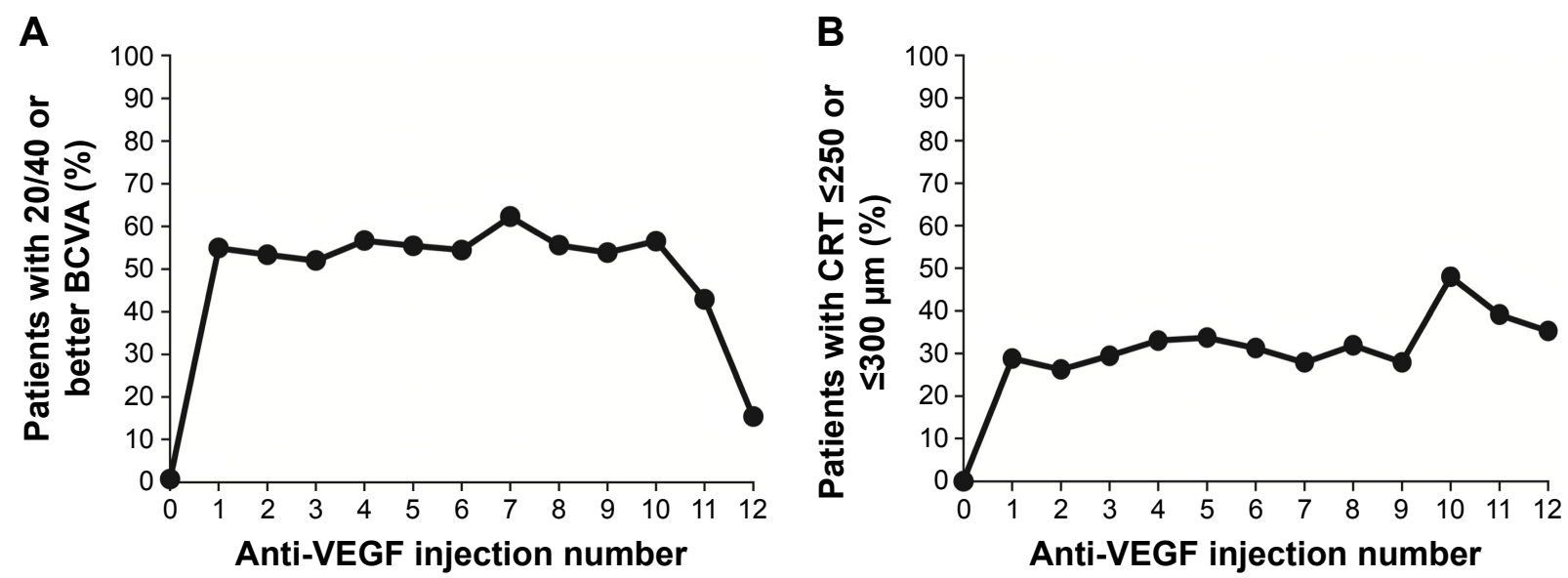

Figure 4 Response rates after each anti-VEGF injection.

Notes: (A) Percentage of patients with 20/40 or better BCVA. The number of patients for injection numbers 0 through I2 was I30, I33, I35, I29, II3, I0I, 79, 53, 45, 39, 23, 14, and 13, respectively. (B) Percentage of patients with CRT $\leq 250 \mu \mathrm{m}$ on TD-OCT or $\leq 300 \mu \mathrm{m}$ on SD-OCT. The number of patients for injection numbers 0 through 12 was I19, II8, 122, I29, 106, 83, 80, 61, 47, 43, 25, 23, and 17, respectively.

Abbreviations: BCVA, best-corrected visual acuity; CRT, central retinal thickness; SD-OCT, spectral-domain optical coherence tomography; TD-OCT, time-domain optical coherence tomography; VEGF, vascular endothelial growth factor.

\section{Ocular treatments and procedures during the study period}

Nearly half of the patients $(44.9 \%)$ received focal laser treatment during the study period (Table 2), and $13.5 \%$ underwent panretinal photocoagulation. A total of 25 intravitreal corticosteroid injections were administered during the study period ( 2 injections of dexamethasone intravitreal implant and 23 injections of triamcinolone acetonide).

Sixteen of the 104 patients who were phakic at the time of their first anti-VEGF injection (15.4\%) had cataract extraction during the study period (Table 2).

\section{Safety outcomes}

Overall, 188 AEs, including 19 AEs related to DME treatment, were reported during the study period. All of the

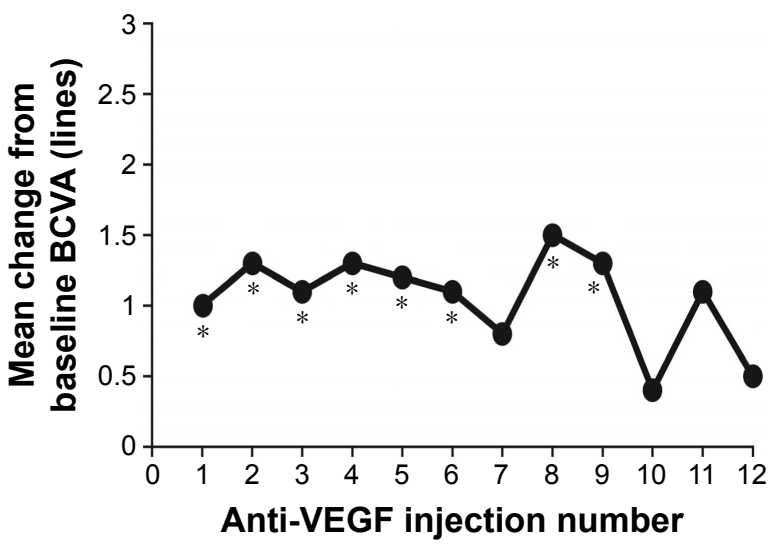

Figure 5 Mean change in BCVA from baseline after each injection.

Notes: The number of patients for injection numbers I through 12 was 114, I17, $109,98,86,69,43,37,33,17,14$, and 10, respectively. $* P \leq 0.038$.

Abbreviations: BCVA, best-corrected visual acuity; VEGF, vascular endothelial growth factor. treatment-related AEs were ocular; the most common were elevated IOP or ocular hypertension/glaucoma (6 reports), ocular pain (3 reports), floaters ( 2 reports), and subconjunctival hemorrhage ( 2 reports). An increase in IOP of $>10 \mathrm{mmHg}$ from baseline was documented in the study eye in 8 patients (5.1\%). Seven of these patients had received only anti-VEGF treatment; the remaining patient had received an injection of intravitreal triamcinolone acetonide, followed by injections of bevacizumab and ranibizumab, before the measured increase in IOP. Twelve patients (7.7\%) had IOP $>25 \mathrm{mmHg}$ and 2 patients $(1.3 \%)$ had IOP $>35 \mathrm{mmHg}$ during the study period. Both of the patients with IOP $>35 \mathrm{mmHg}$ had been treated with bevacizumab only.

\section{Discussion}

In this study, anti-VEGF injections for DME were administered less frequently than the monthly injections recommended in the United States product insert for ranibizumab, and treatment was less effective than in the ranibizumab registration trials. The mean number of injections received by patients was $5.8,5.0$, and 3.4 in the first, second, and third study years, respectively, and the mean BCVA improvement achieved by patients over all injections was 1.1 line (5.5 ETDRS letters). Achievement of the combined primary endpoint was poor: after injections 1 through $9,<25 \%$ of patients who had BCVA and CRT measurements at the same visit achieved both BCVA of 20/40 or better and normal CRT.

The demographic and clinical characteristics of the patients included in the study were typical of patients with DME seen at ophthalmic practices in the United States. The incidence of DME is higher in type 1 than type 2 diabetes, ${ }^{17}$ 
A

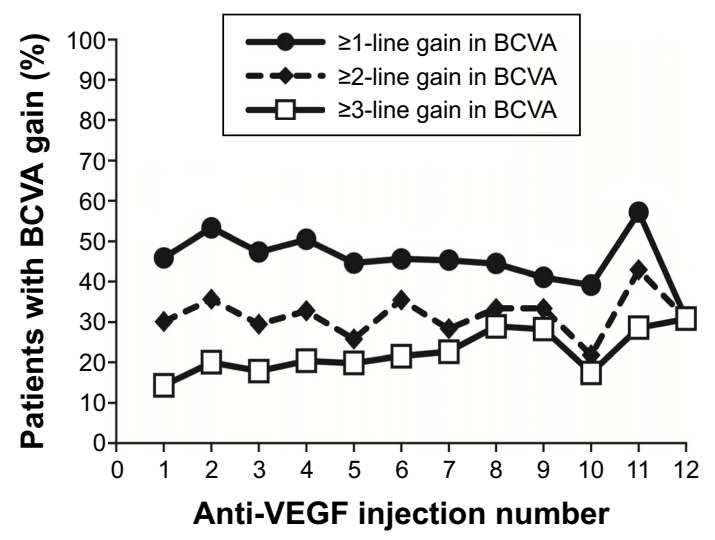

B

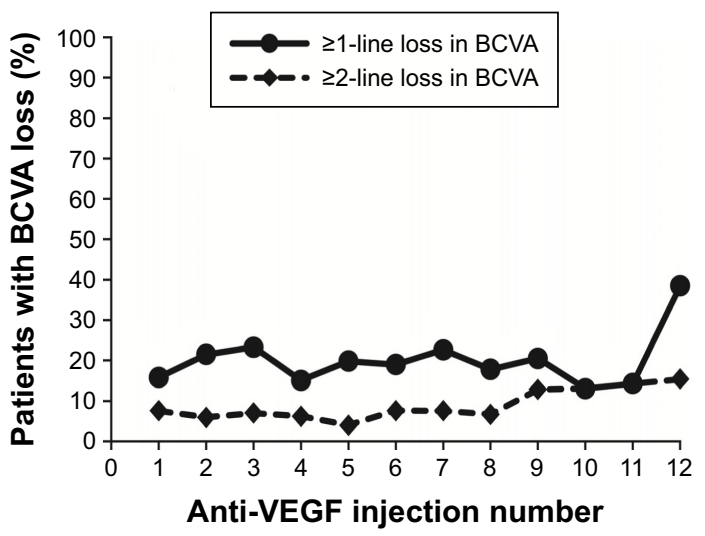

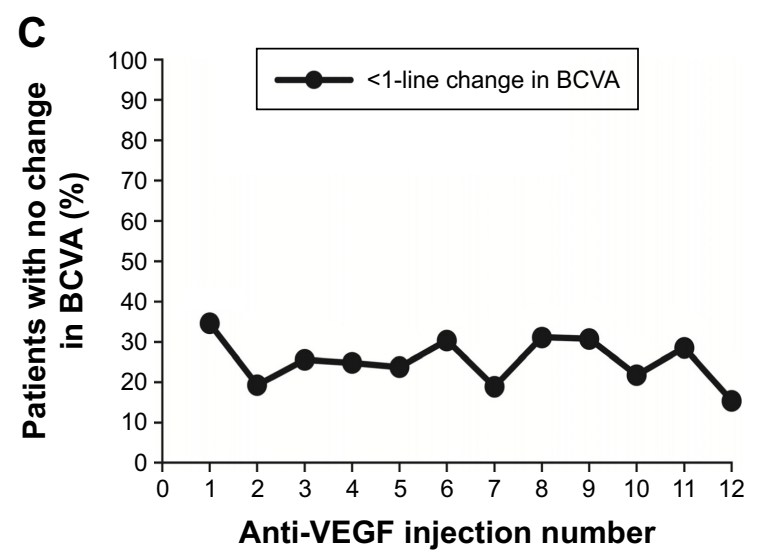

Figure 6 Percentage of patients with (A) gain, (B) loss, or (C) no change in BCVA from baseline after anti-VEGF injection. Note: The number of patients for injection numbers I through 12 was 133, 135, 129, 113, I0I, 79, 53, 45, 39, 23, 14, and I3, respectively. Abbreviations: BCVA, best-corrected visual acuity; VEGF, vascular endothelial growth factor.

yet type 2 diabetes is much more prevalent, and thus, the majority of patients in the study had type 2 diabetes. Patients generally had vision loss (mean BCVA of $~ 20 / 63$ Snellen) as well as retinal thickening (mean CRT of $413 \mu \mathrm{m}$ ) at the time of their first anti-VEGF treatment. The standard of care in DME management includes tight glycemic and blood pressure control. Diabetes management guidelines from the American Diabetes Association recommend $\mathrm{HbA1c}$ testing at least twice each year for diabetic patients with stable glycemic control, and at least 4 times each year for those without stable glycemic control. ${ }^{18}$ However, evidence suggests that at least $30 \%$ of patients with diabetes in the United States do not receive even yearly HbA1c testing, ${ }^{19}$ and in this study, baseline $\mathrm{Hb} 1 \mathrm{Ac}$ data were unavailable for almost $50 \%$ of patients.

At least half of the patients received their first anti-VEGF injection soon after DME diagnosis (median time since diagnosis, 2 weeks), but many patients (40.4\%) had a history of retinal focal laser in the study eye prior to anti-VEGF therapy. Furthermore, $44.9 \%$ of patients received retinal focal laser in the study eye during the study period. An investigation using Medicare claims data from 2008 to 2012 similarly reported that $42.2 \%$ of patients with DME who were treated with anti-VEGF received laser therapy within 1 year after their initial anti-VEGF injection. ${ }^{20}$

There was large variability in the number of anti-VEGF injections that patients received, in part because the period of chart review was variable and patients were required to have only 6 months of data for inclusion in the study. Consequently, the number of patients with 13 or more antiVEGF injections was too small for meaningful analysis of efficacy after the 13th and subsequent injections. Nonetheless, the average interval between anti-VEGF injections was consistently longer than 1 month, and patients who had data available through 1 year received fewer anti-VEGF injections than with optimal monthly administration. ${ }^{13}$ The reasons for the low number of anti-VEGF injections in years 1, 2, and 3 are unclear. Patients with DME have a high burden of health care visits, ${ }^{9}$ and it is possible that missed eye care appointments contributed to reduced injection frequency. For some 


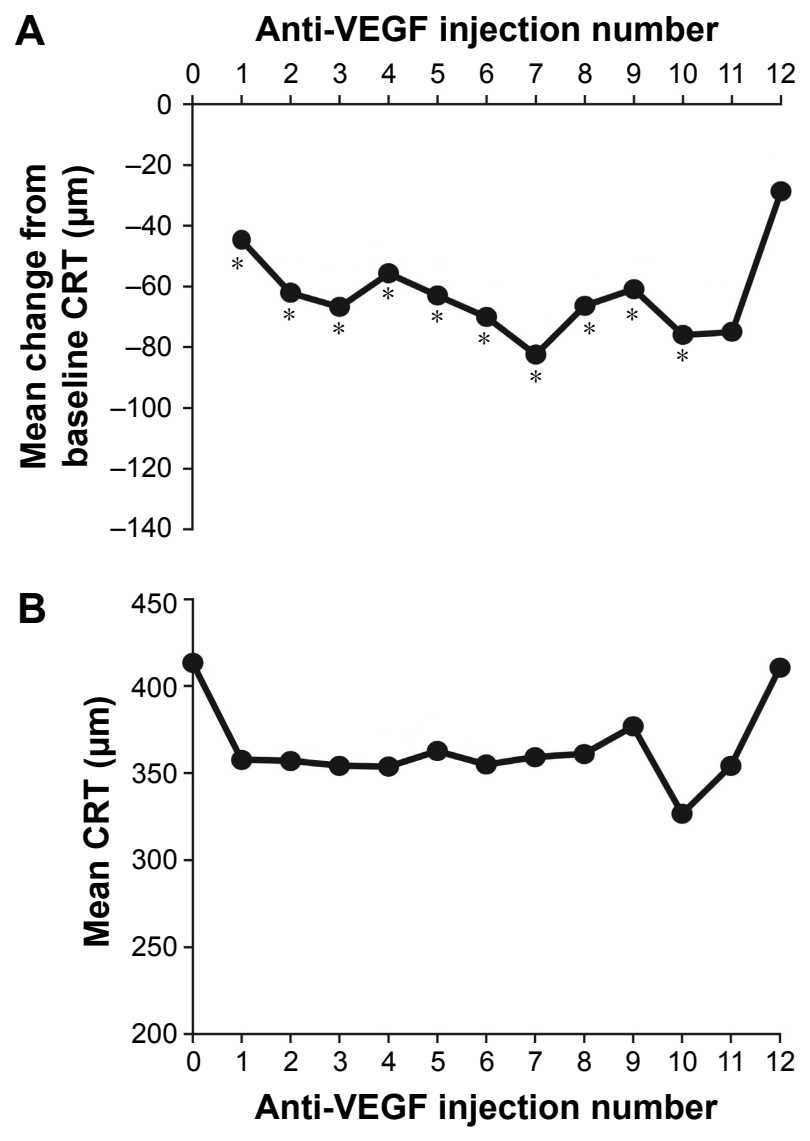

Figure 7 Mean change in CRT from baseline (A) and mean CRT (B) after each injection.

Notes: The number of patients for injection numbers I through 12 in (A) was 95, $95,104,84,68,67,47,37,31,18,13$, and 9 , respectively. The number of patients for injection numbers 0 through 12 in (B) was $119,118,122,129,106,83,80,61$, $47,43,25,23$, and 17 , respectively. $* P \leq 0.015$.

Abbreviations: CRT, central retinal thickness; VEGF, vascular endothelial growth factor.

patients, the number of anti-VEGF injections may have been low because the patients switched to or used other therapy (primarily laser) between anti-VEGF injections. In addition, some patients may have stopped receiving anti-VEGF treatment because of resolution of the DME.

Almost half of the patients received both ranibizumab and bevacizumab during the study period, with the majority of

Table 2 Ocular procedures during the study period

\begin{tabular}{ll}
\hline Procedure, $\mathbf{n}(\%)$ & $\begin{array}{l}\text { Study population } \\
(\mathbf{n}=\mathbf{1 5 6})\end{array}$ \\
\hline Laser photocoagulation & $70(44.9)$ \\
$\quad$ Focal laser & $21(13.5)$ \\
$\quad$ Panretinal photocoagulation & \\
Glaucoma surgeries & $\mathrm{I}(0.6)$ \\
$\quad$ Laser & 0 \\
$\quad$ Incisional & $16(15.4)^{\mathrm{a}}$ \\
\hline
\end{tabular}

Note: apercentage of baseline phakic eyes $(n=104)$ that underwent cataract surgery. the switches in therapy from ranibizumab to bevacizumab. Data on the reasons for switching between anti-VEGF agents were not available for analysis. However, available evidence suggests that ranibizumab demonstrates the same or better efficacy than bevacizumab in the treatment of DME. ${ }^{11,21,22}$ Therefore, switches from ranibizumab to bevacizumab may have been due to the cost of treatment rather than to lack of efficacy of ranibizumab.

The mean gain in BCVA and the percentage of patients with at least 3-line improvement in BCVA in this study were much lower than in the RISE and RIDE ranibizumab registration trials (mean gain of 10.9-12.5 letters and 33.6\%-44.8\% of patients with $\geq 3$-line gain at 24 months with ranibizumab $0.3 \mathrm{mg}$ in RISE/RIDE), ${ }^{10}$ most likely because of the reduced frequency of anti-VEGF treatment. In the RISE and RIDE trials, mean CRT continually improved through 12 months of monthly ranibizumab injections. ${ }^{10}$ In this study, although the mean reduction in CRT appeared to improve somewhat over the first few injections, continual improvement in mean CRT was not seen, possibly because of missing data and the contribution of data after injections by differing subgroups of patients. Despite some fluid resolution, a significant amount of fluid persisted and mean CRT remained substantially elevated ( $\sim 350$ or $360 \mu \mathrm{m}$ on average) after each of the first 10 antiVEGF injections. These results suggest that anti-VEGF as used in clinical practice does not provide optimal outcomes in many patients, ie, a high proportion of patients experience persistent edema despite treatment. The potential consequences of long-term, persistent edema include irreversible damage to vision. For example, in RISE/RIDE, patients who crossed over and received ranibizumab after 2 years of sham injections showed similar improvement in CRT but did not experience the same level of BCVA gain as those treated early (mean gains from baseline after 1 year of ranibizumab treatment were 7.5-7.8 letters versus 10.6-11.1 letters, respectively). ${ }^{23}$ Injection of anti-VEGF more frequently may improve outcomes, but notably, a substantial number of patients in the RISE/RIDE registration trials did not have optimal outcomes even with monthly ranibizumab injections. ${ }^{10}$

Separate analysis of visual and anatomic outcomes showed that DME patients treated with anti-VEGF were more likely to achieve 20/40 BCVA than a dry macula. After the first through ninth anti-VEGF injections, $52 \%-62 \%$ of patients achieved $20 / 40$ or better BCVA, while $26 \%-34 \%$ of patients achieved CRT $\leq 250 \mu \mathrm{m}$ on TD-OCT or $\leq 300 \mu \mathrm{m}$ on SD-OCT. This mismatch between visual and anatomic outcomes suggests that patients do not necessarily have to achieve a dry macula to achieve 20/40 or better BCVA. 
Nevertheless, a dry macula is the optimal goal of treatment, both to maximize visual acuity and to prevent detrimental effects of persistent edema. As an association has been demonstrated between the presence of cystoid DME on OCT and photoreceptor damage, ${ }^{24}$ downstream effects of persistent edema are likely to include additional photoreceptor loss.

Treatment of DME with VEGF inhibitors was well tolerated in the clinical practice setting. There were few reports of AEs related to anti-VEGF or the injection procedure, and there were no reports of thromboembolic events related to anti-VEGF treatment. The lack of complications of antiVEGF treatment seen in this study is consistent with the favorable safety profile of intravitreal VEGF inhibitors demonstrated in clinical trials of anti-VEGF therapy in patients with DME. ${ }^{4-7}$ VEGF inhibitors have become the treatment of choice in DME because of their safety as well as their effectiveness in reducing macular edema and improving visual acuity in many patients.

The main limitation of this study was that the antiVEGF therapies evaluated were limited to ranibizumab and bevacizumab. The chart review included only 1 aflibercept injection because the period of chart review was from June 2010 to September 2014, and aflibercept was not approved for the treatment of DME until July 2014. Some patients may have larger gains in vision with aflibercept than with ranibizumab or bevacizumab treatment. ${ }^{11}$ This study also had the limitations inherent to retrospective studies. In particular, retrospective chart review studies commonly have missing or incomplete data, and in this study, the date of first DME diagnosis was sometimes missing and not always reliable. Finally, patients were required to have received at least 3 anti-VEGF injections to be included in the study. This patient selection bias most likely resulted in improvement in the apparent efficacy and safety of treatment, as patients who do not respond to 1 or 2 injections with reduced edema or improved vision, or who have an adverse reaction to the injection procedure, would be likely to discontinue antiVEGF treatment. An apparent worsening of the efficacy of treatment could also have resulted, if patients received only 1 or 2 anti-VEGF injections with resolution of DME; however, this possibility seems less likely.

The pathogenesis of DME is understood to be complex. ${ }^{25}$ A treatment approach that targets components of the pathogenesis of DME beyond VEGF may be needed for patients with an inadequate response to anti-VEGF. Many inflammatory mediators and processes are involved in the breakdown of the blood-retinal barrier that leads to retinal capillary leakage and the development of DME. ${ }^{26}$ Because corticosteroids have broad anti-inflammatory activity and stabilize the blood-retinal barrier, ${ }^{26}$ patients who are suboptimal responders to anti-VEGF may benefit from the addition of intravitreal corticosteroid injections as well as from use of adjunctive laser.

\section{Conclusion}

The results of this study confirm that patients with DME treated with intravitreal anti-VEGF in the clinical practice setting receive injections less frequently than in reported clinical trials. Many patients treated with ranibizumab and bevacizumab did not achieve 20/40 or better visual acuity and/or a dry macula after anti-VEGF injection. The results indicate that a substantial number of patients with DME do not respond optimally to anti-VEGF therapy. Patients who are suboptimal responders to anti-VEGF treatment may need additional therapies, including intravitreal corticosteroid and laser therapy.

\section{Acknowledgments}

This study was sponsored by Allergan plc, Dublin, Ireland. A contract research organization, Trial Runners, LLC (Dickinson, ND, USA), was responsible for project and data management and performed the statistical analysis of the data. Writing and editorial assistance was provided to the authors by Kate Ivins, PhD, Evidence Scientific Solutions, Philadelphia, PA, USA, and funded by Allergan plc. All authors met the ICMJE authorship criteria. Neither honoraria nor payments were made for authorship. This study was presented in part at the American Society of Retina Specialists (ASRS) Annual Meeting, Vienna, Austria, July 12, 2015. ECHO Study Group Principal Investigators are as follows: Kevin J Blinder, MD (St Louis, MO, USA); David S Boyer, MD (Beverly Hills, CA, USA); Antonio Capone Jr, MD (Royal Oak, MI, USA); Sanford Chen, MD (Santa Ana, CA, USA); Pravin Dugel, MD (Phoenix, AZ, USA); J Michael Jumper, MD (San Francisco, CA, USA); Alan Margherio, MD (Grand Rapids, MI, USA); Joel Pearlman, MD (Sacramento, CA, USA); Michael A Singer, MD (San Antonio, TX, USA).

\section{Disclosure}

The authors have no proprietary interest in this work. Financial arrangements of the authors with companies whose products may be related to the present report are listed below, as declared by the authors. KJ Blinder is a consultant to Allergan, Bausch + Lomb, Dutch Ophthalmics, Genentech, Regeneron, Synergetics, and Thrombogenics and 
has received lecture fees from Allergan, Bausch + Lomb, Regeneron, Synergetics, and Thrombogenics. PU Dugel is a consultant to Allergan and has received research grants from Allergan. S Chen is a consultant to Alcon, Alimera, Allergan, Genentech, and Regeneron. JM Jumper is a clinical investigator for Allergan, Genentech, Ophthotech, and Regeneron and has received research grants from Allergan. JG Walt is an employee of Allergan. DA Hollander and LC Scott are former employees of Allergan. The authors report no other conflicts of interest in this work.

\section{References}

1. Arevalo JF. Diabetic macular edema: current management 2013. World J Diabetes. 2013;4(6):231-233.

2. American Diabetes Association. Standards of medical care in diabetes 2009. Diabetes Care. 2009;32(suppl 1):S13-S61.

3. Boyer DS, Hopkins JJ, Sorof J, Ehrlich JS. Anti-vascular endothelial growth factor therapy for diabetic macular edema. Ther Adv Endocrinol Metab. 2013;4(6):151-169.

4. Nguyen QD, Shah SM, Heier JS, et al; READ-2 Study Group. Primary end point (six months) results of the Ranibizumab for Edema of the mAcula in Diabetes (READ-2) study. Ophthalmology. 2009;116(11): 2175-2181.e1.

5. Mitchell P, Bandello F, Schmidt-Erfurth U, et al; RESTORE Study Group. The RESTORE study: ranibizumab monotherapy or combined with laser versus laser monotherapy for diabetic macular edema. Ophthalmology. 2011;118(4):615-625.

6. Michaelides M, Kaines A, Hamilton RD, et al. A prospective randomized trial of intravitreal bevacizumab or laser therapy in the management of diabetic macular edema (BOLT study) 12-month data: report 2. Ophthalmology. 2010;117(6):1078-1086.e2.

7. Brown DM, Schmidt-Erfurth U, Do DV, et al. Intravitreal aflibercept for diabetic macular edema: 100-week results from the VISTA and VIVID studies. Ophthalmology. 2015;122(10):2044-2052.

8. Shea AM, Curtis LH, Hammill BG, et al. Resource use and costs associated with diabetic macular edema in elderly persons. Arch Ophthalmol. 2008;126(12):1748-1754.

9. Wallick CJ, Hansen RN, Campbell J, Kiss S, Kowalski JW, Sullivan SD. Comorbidity and health care resource use among commercially insured non-elderly patients with diabetic macular edema. Ophthalmic Surg Lasers Imaging Retina. 2015;46(7):744-751.

10. Nguyen QD, Brown DM, Marcus DM, et al. Ranibizumab for diabetic macular edema: results from 2 phase III randomized trials: RISE and RIDE. Ophthalmology. 2012;119(4):789-801.

11. Diabetic Retinopathy Clinical Research Network, Wells JA, Glassman AR, et al. Aflibercept, bevacizumab, or ranibizumab for diabetic macular edema. N Engl J Med. 2015;372(13):1193-1203.
12. Wells JA, Glassman AR, Ayala AR, et al; Diabetic Retinopathy Clinical Research Network. Aflibercept, bevacizumab, or ranibizumab for diabetic macular edema: two-year results from a comparative effectiveness randomized clinical trial. Ophthalmology. 2016;123(6):1351-1359.

13. Kiss S, Liu Y, Brown J, et al. Clinical utilization of anti-vascular endothelial growth-factor agents and patient monitoring in retinal vein occlusion and diabetic macular edema. Clin Ophthalmol. 2014;8: 1611-1621.

14. Holekamp NM, Liu Y, Yeh WS, et al. Clinical utilization of anti-VEGF agents and disease monitoring in neovascular age-related macular degeneration. Am J Ophthalmol. 2014;157(4):825-833.e1.

15. VanderBeek BL, Shah N, Parikh PC, Ma L. Trends in the care of diabetic macular edema: analysis of a national cohort. PLoS One. 2016;11(2): e0149450.

16. Gregori NZ, Feuer W, Rosenfeld PJ. Novel method for analyzing Snellen visual acuity measurements. Retina. 2010;30(7):1046-1050.

17. Lee R, Wong TY, Sabanayagam C. Epidemiology of diabetic retinopathy, diabetic macular edema and related vision loss. Eye Vis (Lond). 2015;2:17

18. American Diabetes Association. Standards of medical care in diabetes 2014. Diabetes Care. 2014;37(suppl 1):S14-S80.

19. Chen R, Cheadle A, Johnson D, Duran B. US trends in receipt of appropriate diabetes clinical and self-care from 2001 to 2010 and racial/ ethnic disparities in care. Diabetes Educ. 2014;40(6):756-766.

20. Jiang S, Barner JC, Park C, Ling YL. Treatment patterns of anti-vascular endothelial growth factor and laser therapy among patients with diabetic macular edema. J Manag Care Spec Pharm. 2015;21(9):735-741.

21. Ehrlich R, Dan I, Deitch I, Axer-Siegel R, Mimouni K. The effectiveness of intravitreal ranibizumab in patients with diabetic macular edema who have failed to respond to intravitreal bevacizumab. Ophthalmologica. 2016;235(3):133-136.

22. Wiley HE, Thompson DJ, Bailey C, et al. A crossover design for comparative efficacy: a 36-week randomized trial of bevacizumab and ranibizumab for diabetic macular edema. Ophthalmology. 2016;123(4): 841-849.

23. Brown DM, Nguyen QD, Marcus DM, et al; RIDE and RISE Research Group. Long-term outcomes of ranibizumab therapy for diabetic macular edema: the 36-month results from two phase III trials: RISE and RIDE. Ophthalmology. 2013;120(10):2013-2022.

24. Murakami T, Nishijima K, Akagi T, et al. Optical coherence tomographic reflectivity of photoreceptors beneath cystoid spaces in diabetic macular edema. Invest Ophthalmol Vis Sci. 2012;53(3):1506-1511.

25. Zhang X, Zeng H, Bao S, Wang N, Gillies MC. Diabetic macular edema: new concepts in patho-physiology and treatment. Cell Biosci. 2014;4:27.

26. Dugel PU, Bandello F, Loewenstein A. Dexamethasone intravitreal implant in the treatment of diabetic macular edema. Clin Ophthalmol. 2015;9:1321-1335.
Clinical Ophthalmology

\section{Publish your work in this journal}

Clinical Ophthalmology is an international, peer-reviewed journal covering all subspecialties within ophthalmology. Key topics include: Optometry; Visual science; Pharmacology and drug therapy in eye diseases; Basic Sciences; Primary and Secondary eye care; Patient Safety and Quality of Care Improvements. This journal is indexed on Submit your manuscript here: http://www.dovepress.com/clinical-ophthalmology-journal

\section{Dovepress}

PubMed Central and CAS, and is the official journal of The Society of Clinical Ophthalmology (SCO). The manuscript management system is completely online and includes a very quick and fair peer-review system, which is all easy to use. Visit http://www.dovepress.com/ testimonials.php to read real quotes from published authors. 